\title{
On some topological properties of intuitionistic 2-fuzzy $n$-normed linear spaces
}

\author{
Lj.D.R. Kočinac*1 (D), V.A. Khan ${ }^{2}$ (D), K.M.A.S. Alshlool ${ }^{2}$ (D) H. Altaf ${ }^{2}$ (D) \\ ${ }^{1}$ University of Niš, Faculty of Sciences and Mathematics, 18000 Niš, Serbia \\ ${ }^{2}$ Aligarh Muslim University, Department of Mathematics, Aligarh 202002, India
}

\begin{abstract}
Motivated by the notion of $n$-norm due to Gähler, in this article we define the concept of intuitionistic 2-fuzzy $n$-normed space in general setting of $t$-norm as a generalization of intuitionistic fuzzy normed space in the sense of Bag and Samanta. Further we define the notion of $\alpha$-n-norm corresponding to intuitionistic 2-fuzzy $n$-norm. In addition, we discuss some basic properties of convergence and completeness for intuitionistic 2-fuzzy $n$-normed spaces.
\end{abstract}

Mathematics Subject Classification (2010). 03E72, 40A05, 46B20, 46S40, 54A20

Keywords. fuzzy set, intuitionistic fuzzy set, 2 -fuzzy $n$-normed space, $t$-norm, $t$-conorm

\section{Introduction}

A lot of significant developments of Zadeh's theory of fuzzy sets [21] have been taken place to figure out the fuzzy analogues of the classical set theory. Noticeably, the area of fuzzy set study has become the focus of many researchers for the last 50 years. It was applied very actively in the field of science and engineering, such as computer programming [8] and nonlinear dynamical systems [10]. Once we talk about the fuzzy set theory, we get to put the light on the success of Atanassov [1,2] who introduced the concept of intuitionistic fuzzy sets. After that, Çoker [5] studied intuitioniostic topological spaces, while Park [15] introduced and studied the concept of intuitionistic fuzzy metric space. Saadati and Park [18] introduced the concept of intuitionistic fuzzy normed space. On the other side, Gähler [6,7] has introduced and developed the satisfactory theory of 2norms and $n$-norms on a linear space. After that, many authors motivated by Gähler's work, have generalized and developed the idea of intuitionistic fuzzy normed space to intuitionistic fuzzy 2-normed spaces (see, for example, [13]) and investigated some of their topological properties. Later, Bag and Samanta [4] redefined the notion of intuitionistic fuzzy normed space in such a way that the underlying $t$-norm and $t$-conorm are considered in general setting in the sense that only continuity of $t$-norm and $t$-conorm at $(1,1)$ and $(0,0)$, respectively, are used. This article develops and supports this theory as in some cases the ordinary norms do not work. The concepts of fuzzy norm and $\alpha$-norm were

\footnotetext{
* Corresponding Author.

Email addresses: lkocinac@gmail.com (Lj.D.R. Kočinac), vakhanmaths@gmail.com (V.A. Khan), k_moto@yahoo.com (K.M.A.S. Alshlool), vakhanmaths@gmail.com (H. Altaf)

Received: 08.12.2017; Accepted: 19.10.2018
} 
introduced by Bag and Samanta in [3]. The concept of fuzzy $n$-normed linear spaces has been studied by many authors like [11,14,17]. In 2012, Park and Alaca [16] introduced the concept of 2 -fuzzy $n$-normed linear space or fuzzy $n$-normed linear space of the set of all fuzzy sets of a non-empty set. These authors defined the notion of $\alpha$-n-norms on a linear space corresponding to the fuzzy $n$-norm by using some ideas from [20].

Our main concern here is to define the concept of intuitionistic 2-fuzzy $n$-normed space in general $t$-norm as a generalization of intuitionistic fuzzy normed spaces due to Saadati and Park [18], but in the sense of Bag and Samanta [4]. Further we define the notion of $\alpha$ $n$-norms corresponding to intuitionistic 2-fuzzy $n$-norm. In addition, we discuss some basic properties of convergence and completeness for intuitionistic 2-fuzzy $n$-normed spaces.

Throughout the article $\mathbb{N}, \mathbb{R}$ and $\mathbb{C}$ will be the sets of natural, real and complex numbers, respectively. By $\mathbb{K}$ we denote the field of real or complex numbers.

\section{Definitions and preliminaries}

In this section, we present some preliminary definitions and results related to $n$-normed spaces and 2-fuzzy $n$-normed spaces used in this article.

Definition 2.1 ([9], [12]). Let $n \in \mathbb{N}$ and let $X$ be a real linear space of dimension $d \geq n$. A real-valued function $\|\cdot, \ldots, \cdot\|$ on $X^{n}$ satisfying the following properties:

(i) $\left\|x_{1}, x_{2}, \ldots, x_{n}\right\|=0$ if and only if $x_{1}, x_{2}, \ldots, x_{n}$ are linearly dependent,

(ii) $\left\|x_{1}, x_{2}, \ldots, x_{n}\right\|$ is invariant under any permutation,

(iii) $\left\|x_{1}, x_{2}, \ldots, \alpha x_{n}\right\|=|\alpha|\left\|x_{1}, x_{2}, \ldots, x_{n}\right\|$ for any $\alpha \in \mathbb{R}$,

(iv) $\left\|x_{1}, x_{2}, \ldots, x_{n-1}, y+z\right\| \leq\left\|x_{1}, x_{2}, \ldots, x_{n-1}, y\right\|+\left\|x_{1}, x_{2}, \ldots, x_{n-1}, z\right\|$

is called an $n$-norm on $X$, and the pair $(X,\|\cdot, \ldots, \cdot\|)$ is called an $n$-normed linear space.

Definition 2.2. Let $X$ be a linear space over $\mathbb{K}$ and $F(X)$ be the set of all fuzzy sets in $X$. If $f \in F(X)$, then $f=\{(x, \mu): x \in X, \mu \in(0,1]\}$. Clearly, $f$ is a bounded function $(|f(x)| \leq 1)$. Then $F(X)$ is a linear space over the field $\mathbb{K}$, where the addition and scalar multiplication are defined by

$$
f+g=\{(x, \mu)+(y, \nu)\}=\{(x+y, \mu \wedge \nu):(x, \mu) \in f,(y, \nu) \in g\},
$$

and

$$
\lambda f=\{(\lambda x, \mu):(x, \mu) \in f\}, \lambda \in \mathbb{K} .
$$

The linear space $F(X)$ is said to be a normed linear space if for every $f \in F(X)$ there is associated a non-negative real number $\|f\|$ (called the norm of $f$ ) in such a way that

(1) $\|f\|=0$ if and only if $f=0$,

(2) $\|\lambda f\|=|\lambda|\|f\|, \lambda \in \mathbb{K}$,

(3) $\|f+g\| \leq\|f\|+\|g\|$ for every $f, g \in F(X)$.

Then $(F(X),\|\cdot\|)$ is a normed linear space.

Definition 2.3 ([20]). A fuzzy set on $F(X)$ is called a 2-fuzzy set on $X$.

Definition 2.4 ([15]). Let $X$ be a real linear space of dimension $d \geq n, n \in \mathbb{N}$, and $F(X)$ be the set of all fuzzy sets in $X$. Assume that a $[0,1]$-valued function $\|\cdot, \ldots, \cdot\|$ on $F(X)^{n}$ satisfying the following properties:

(i) $\left\|f_{1}, f_{2}, \ldots, f_{n}\right\|=0$ if and only if $f_{1}, f_{2}, \ldots, f_{n}$ are linearly dependent;

(ii) $\left\|f_{1}, f_{2}, \ldots, f_{n}\right\|$ is invariant under any permutation;

(iii) $\left\|f_{1}, f_{2}, \ldots, \lambda f_{n}\right\|=|\lambda|\left\|f_{1}, f_{2}, \ldots, f_{n}\right\|$ for any $\lambda \in \mathbb{K}$;

(iv) $\left\|f_{1}, f_{2}, \ldots, f_{n-1}, y+z\right\| \leq\left\|f_{1}, x_{2}, \ldots, f_{n-1}, y\right\|+\left\|f_{1}, f_{2}, \ldots, f_{n-1}, z\right\|$.

Then $(F(X),\|\cdot, \ldots, \cdot\|)$ is an $n$-normed linear space or $(X,\|\cdot, \ldots, \cdot\|)$ is a 2 -n-normed linear space. 
Definition 2.5 ([14]). Let $F(X)$ be a real linear space. A fuzzy subset $N$ of $F(X)^{n} \times \mathbb{R}$ is called a 2-fuzzy n-norm on $X$ (or a fuzzy n-norm on $F(X)$ ) if

(2-N1): for all $t \in \mathbb{R}$, with $t \leq 0, N\left(f_{1}, f_{2}, \ldots, f_{n}, t\right)=0$,

(2-N2): for all $t \in \mathbb{R}$, with $t>0, N\left(f_{1}, f_{2}, \ldots, f_{n}, t\right)=1$ if and only if $f_{1}, f_{2}, \ldots, f_{n}$ are linearly dependent,

(2-N3): $N\left(f_{1}, f_{2}, \ldots, f_{n}, t\right)$ is invariant under any permutation of $f_{1}, f_{2}, \ldots, f_{n}$,

(2-N4): for all $t \in \mathbb{R}$, with $t>0, N\left(f_{1}, f_{2}, \ldots, \lambda f_{n}, t\right)=N\left(f_{1}, f_{2}, \ldots, f_{n}, \frac{t}{|\lambda|}\right)$ if $\lambda \neq 0$, $\lambda \in \mathbb{K}$,

(2-N5): for all $s, t \in \mathbb{R}, N\left(f_{1}, \ldots, f_{n}+f_{n}^{\prime}, s+t\right) \geq \min \left\{N\left(f_{1}, \ldots, f_{n}, s\right), N\left(f_{1}, \ldots, f_{n}^{\prime}, t\right)\right\}$,

$(2-\mathrm{N} 6): N\left(f_{1}, f_{2}, \ldots, f_{n}, \cdot\right):(0, \infty) \rightarrow[0,1]$ is continuous,

$(2-\mathrm{N} 7): \lim _{t \rightarrow \infty} N\left(f_{1}, f_{2}, \ldots, f_{n}, t\right)=1$.

Then $(F(X), N)$ is a fuzzy $n$-normed linear space or $(X, N)$ is a 2-fuzzy $n$-normed linear space.

Remark 2.6 ([15]). The non-decreasing property of $N\left(f_{1}, f_{2}, \ldots, f_{n}, \cdot\right)$ in a 2 -fuzzy $n$ normed linear space $(X, N)$ follows from $(2-\mathrm{N} 4)$ and (2-N5) for all $f_{1}, f_{2}, \ldots, f_{n} \in F(X)$.

Definition $2.7([19])$. A binary operation $*:[0,1] \times[0,1] \rightarrow[0,1]$ is said to be continuous t-norm, if the following hold:

(i) $*$ is associative and commutative,

(ii) $*$ is continuous,

(iii) $x * 1=x$ for all $x \in[0,1]$,

(iv) $x * y \leq z * w$ whenever $x \leq z$ and $y \leq w$, where $x, y, z, w \in[0,1]$.

Definition 2.8 ([19]). A binary operation $\diamond:[0,1] \times[0,1] \rightarrow[0,1]$ is said to be continuous $t$-conorm if it satisfies the following properties:

(i) $\diamond$ is associative and commutative,

(ii) $\diamond$ is continuous,

(iii) $x \diamond 0=x$ for all $x \in[0,1]$,

(iv) $x \diamond y \leq z \diamond w$ whenever $x \leq z$ and $y \leq w$, where $x, y, z, w \in[0,1]$.

Remark $2.9([15]) . \quad$ (i) For any $r_{1}, r_{2} \in(0,1)$ with $r_{1}>r_{2}$ there exist $r_{3}, r_{4} \in(0,1)$ such that $r_{1} * r_{3} \geq r_{2}$ and $r_{1} \geq r_{4} \diamond r_{2}$

(ii) For any $r_{5} \in(0,1)$ there exist $r_{6}, r_{7} \in(0,1)$ such that $r_{6} * r_{6} \geq r_{5}$ and $r_{7} \diamond r_{7} \leq r_{5}$.

Definition 2.10 ([1]). Let $E$ be any set. An intuitionistic fuzzy set $A$ of $E$ is an object of the form $A=\left\{\left(x, \mu_{A}(x), \nu_{A}(x)\right): x \in E\right\}$, where the functions $\mu: E \rightarrow[0,1]$ and $\nu: E \rightarrow[0,1]$ denote the degree of membership and the non-membership of the element $x \in E$, respectively, and $0 \leq \mu_{A}(x)+\nu_{A}(x) \leq 1$.

\section{Intuitionistic 2-fuzzy $n$-normed linear spaces}

In this section we define a new and interesting notion of intuitionisitic 2 -fuzzy $n$-normed linear space.

Definition 3.1. Let $F(X)$ be a linear space over a field $\mathbb{K}$ of dimension $d \geq n$, * a continuous $t$-norm, $\diamond$ a continuous $t$-conorm. An intuitionisitic 2-fuzzy $n$-norm, for short I2FnN, on $X$ (or intuitionisitic fuzzy $n$-norm on $F(X)$, for short IFnN) is an object of the form

$$
A=\left\{F(X), \mu\left(f_{1}, \ldots, f_{n}, t\right), \nu\left(f_{1}, \ldots, f_{n}, t\right):\left(f_{1}, \ldots, f_{n}, t\right) \in[F(X)]^{n} \times \mathbb{R}\right\},
$$

where $\mu$ and $\nu$ are fuzzy sets on $[F(X)]^{n} \times \mathbb{R}, \mu$ denote the degree of membership, and $\nu$ denote the degree of non-membership of $\left(f_{1}, f_{2}, \ldots, f_{n}, t\right) \in[F(X)]^{n} \times \mathbb{R}$ satisfying the following conditions:

(I2FnN1): for all $t \in \mathbb{R}$ with $t \leq 0, \mu\left(f_{1}, f_{2}, \ldots, f_{n}, t\right)=0$, 
(I2FnN2): for all $t \in \mathbb{R}$ with $t>0, \mu\left(f_{1}, f_{2}, \ldots, f_{n}, t\right)=1$ if and only if $f_{1}, f_{2}, \ldots, f_{n}$ are linearly dependent,

(I2FnN3): $\mu\left(f_{1}, f_{2}, \ldots, f_{n}, t\right)$ is invariant under any permutation of $f_{1}, f_{2}, \ldots, f_{n}$,

(I2FnN4): for all $c \in \mathbb{K}$ with $c \neq 0, \mu\left(f_{1}, f_{2}, \ldots, c f_{n}, t\right)=\mu\left(f_{1}, f_{2}, \ldots, f_{n}, t /|c|\right)$,

(I2FnN5): for all $\left.s, t \in \mathbb{R}, \mu\left(f_{1}, \ldots, f_{n}+f_{n}^{\prime}, s+t\right) \geq \mu\left(f_{1}, \ldots, f_{n}, s\right) * \mu\left(f_{1}, \ldots, f_{n}^{\prime}, t\right)\right\}$, (I2FnN6): $\lim _{t \rightarrow \infty} \mu\left(f_{1}, f_{2}, \ldots, f_{n}, t\right)=1$,

(I2FnN7): for all $t \in \mathbb{R}$ with $t \leq 0, \nu\left(f_{1}, f_{2}, \ldots, f_{n}, t\right)=1$,

(I2FnN8): for all $t \in \mathbb{R}$ with $t>0, \nu\left(f_{1}, f_{2}, \ldots, f_{n}, t\right)=0$ if and only if $f_{1}, f_{2}, \ldots, f_{n}$ are linearly dependent,

(I2FnN9): $\nu\left(f_{1}, f_{2}, \ldots, f_{n}, t\right)$ is invariant under any permutation of $f_{1}, f_{2}, \ldots, f_{n}$,

(I2FnN10): for all $c \in \mathbb{K}$ with $c \neq 0, \nu\left(f_{1}, f_{2}, \ldots, c f_{n}, t\right)=\nu\left(f_{1}, f_{2}, \ldots, f_{n}, t /|c|\right)$,

(I2FnN11): for all $\left.s, t \in \mathbb{R}, \nu\left(f_{1}, \ldots, f_{n}+f_{n}^{\prime}, s+t\right) \leq \nu\left(f_{1}, \ldots, f_{n}, s\right) \diamond \nu\left(f_{1}, \ldots, f_{n}^{\prime}, t\right)\right\}$,

(I2FnN12): $\lim _{t \rightarrow \infty} \nu\left(f_{1}, f_{2}, \ldots, f_{n}, t\right)=0$.

In this case $(\mu, \nu)$ is called an intuitionistic 2-fuzzy $n$-norm on $X$ or intuitionistic fuzzy $n$-norm on $F(X)$ and we denote it by $(\mu, \nu)_{n}$. Then the five-tuple $(X, \mu, \nu, *, \diamond)$ is called an intuitionisitic 2-fuzzy n-normed linear space (for short I2FnNLS) or $(F(X), \mu, \nu, *, \diamond)$ is called an intuitionisitic fuzzy $n$-normed linear space (for short IFnNLS).

Remark 3.2. The non-decreasing property of $\mu\left(f_{1}, f_{2}, \ldots, f_{n}, t\right)$ follows from (I2FN2) and (I2FN5), and the non-increasing property of $\nu\left(f_{1}, f_{2}, \ldots, f_{n}, t\right)$ follows from (I2FN8) and (I2FN11).

Hereafter we use the notion intuitionisitic fuzzy $n$-norm on $F(X)$ instead of intuitionisitic 2-fuzzy $n$-norm on $X$.

We construct the following example of an intuitionisitic fuzzy $n$-normed linear space.

Example 3.3. Let $(F(X),\|\cdot, \cdots\|)$ be an 2-fuzzy $n$-normed linear space, $a * b=\min \{a, b\}$ and $a \diamond b=\max \{a, b\}$, for all $a, b \in[0,1], t \in \mathbb{R}$, and $f_{1}, f_{2}, \cdots, f_{n} \in F(X)$. Define

$$
\mu\left(f_{1}, f_{2}, \ldots, f_{n}, t\right)= \begin{cases}\frac{t}{t+\left\|f_{1}, f_{2}, \ldots, f_{n}\right\|}, & \text { if } t>0, \\ 0, & t \leq 0,\end{cases}
$$

and

$$
\nu\left(f_{1}, f_{2}, \ldots, f_{n}, t\right)= \begin{cases}\frac{\left\|f_{1}, f_{2}, \ldots, f_{n}\right\|}{t+\left\|f_{1}, f_{2}, \ldots, f_{n}\right\|}, & \text { if } t>0, \\ 1, & t \leq 0 .\end{cases}
$$

If $A=\left\{F(X), N\left(f_{1}, \ldots, f_{n}, t\right), M\left(f_{1}, \ldots, f_{n}, t\right): f_{1}, \ldots, f_{n} \in F(X)\right\}$, then $(F(X), \mu, \nu, *, \diamond)$ is an intuitionistic fuzzy $n$-normed linear space.

Proof. We prove that (IFnN1)-(IFnN12) are satisfied.

(IFnN1) For all $t \in \mathbb{R}$ with $t \leq 0, \mu\left(f_{1}, f_{2}, \ldots, f_{n}, t\right)=0$.

(IFnN2)] for all $t \in \mathbb{R}$ with $t>0$, we have

$$
\begin{aligned}
\mu\left(f_{1}, f_{2}, \ldots, f_{n}, t\right)=1 & \Leftrightarrow \frac{t}{t+\left\|f_{1}, f_{2}, \ldots, f_{n}\right\|}=1 \\
& \Leftrightarrow t=t+\left\|f_{1}, f_{2}, \ldots, f_{n}\right\| \\
& \Leftrightarrow\left\|f_{1}, f_{2}, \ldots, f_{n}\right\|=0 \\
& \Leftrightarrow f_{1}, f_{2}, \ldots, f_{n} \text { are linearly independent. }
\end{aligned}
$$

(IFnN3) For all $t \in \mathbb{R}$ with $t>0$, we have

$$
\begin{aligned}
\mu\left(f_{1}, f_{2}, \ldots, f_{n}, t\right) & =\frac{t}{t+\left\|f_{1}, f_{2}, \ldots, f_{n}\right\|}=\frac{t}{t+\left\|f_{1}, f_{2}, \ldots, f_{n}, f_{n-1}\right\|} \\
& =\mu\left(f_{1}, f_{2}, \ldots, f_{n}, f_{n-1}, t\right)=\ldots
\end{aligned}
$$


(IFnN4) For all $t \in \mathbb{R}$ with $t>0$ and $c \in \mathbb{K}$,

$$
\begin{aligned}
\mu\left(f_{1}, f_{2}, \ldots, f_{n}, t /|c|\right) & =\frac{t /|c|}{t /|c|+\| f_{1}, f_{2}, \ldots, f_{n}} \| \\
& =\frac{t}{t+|c|\left\|f_{1}, f_{2}, \ldots, f_{n}\right\|}=\frac{t}{t+\left\|f_{1}, f_{2}, \ldots, c f_{n}\right\|} \\
& =\mu\left(f_{1}, f_{2}, \ldots, c f_{n}, t\right) .
\end{aligned}
$$

(IFnN5) Without of loss of generality assume that

$$
\begin{gathered}
\mu\left(f_{1}, f_{2}, \ldots, f_{n}^{\prime}, t\right) \leq \mu\left(f_{1}, f_{2}, \ldots, f_{n}, s\right) \\
\Rightarrow \frac{t}{t+\left\|f_{1}, f_{2}, \ldots, f_{n}^{\prime}\right\|} \leq \frac{s}{s+\left\|f_{1}, f_{2}, \ldots, f_{n}\right\|} \\
\Rightarrow t\left(s+\left\|f_{1}, f_{2}, \ldots, f_{n}\right\|\right) \leq s\left(t+\left\|f_{1}, f_{2}, \ldots, f_{n}^{\prime}\right\|\right) \\
\Rightarrow t\left\|f_{1}, f_{2}, \ldots, f_{n}\right\| \leq s\left\|f_{1}, f_{2}, \ldots, f_{n}^{\prime}\right\| \\
\Rightarrow\left\|f_{1}, f_{2}, \ldots, f_{n}\right\| \leq \frac{s}{t}\left\|f_{1}, f_{2}, \ldots, f_{n}^{\prime}\right\| .
\end{gathered}
$$

Therefore,

$$
\begin{aligned}
\left\|f_{1}, f_{2}, \ldots, f_{n}\right\| & +\left\|f_{1}, f_{2}, \ldots, f_{n}^{\prime}\right\| \leq \frac{s}{t}\left\|f_{1}, f_{2}, \ldots, f_{n}^{\prime}\right\|+\left\|f_{1}, f_{2}, \ldots, f_{n}^{\prime}\right\| \\
& \leq\left(\frac{s}{t}+1\right)\left\|f_{1}, f_{2}, \ldots, f_{n}^{\prime}\right\|=\left(\frac{s+t}{t}\right)\left\|f_{1}, f_{2}, \ldots, f_{n}^{\prime}\right\| .
\end{aligned}
$$

But,

$$
\begin{aligned}
\left\|f_{1}, f_{2}, \ldots, f_{n}+f_{n}^{\prime}\right\| & \leq\left\|f_{1}, f_{2}, \ldots, f_{n}\right\|+\left\|f_{1}, f_{2}, \ldots, f_{n}^{\prime}\right\| \\
& \leq\left(\frac{s+t}{t}\right)\left\|f_{1}, f_{2}, \ldots, f_{n}^{\prime}\right\| \\
\Rightarrow \frac{\left\|f_{1}, f_{2}, \ldots, f_{n}+f_{n}^{\prime}\right\|}{s+t} & \leq \frac{\left\|f_{1}, f_{2}, \ldots, f_{n}^{\prime}\right\|}{t} \\
\Rightarrow 1+\frac{\left\|f_{1}, f_{2}, \ldots, f_{n}+f_{n}^{\prime}\right\|}{s+t} & \leq 1+\frac{\left\|f_{1}, f_{2}, \ldots, f_{n}^{\prime}\right\|}{t} \\
\Rightarrow \frac{s+t+\left\|f_{1}, f_{2}, \ldots, f_{n}+f_{n}^{\prime}\right\|}{s+t} & \leq \frac{t+\left\|f_{1}, f_{2}, \ldots, f_{n}^{\prime}\right\|}{t} \\
\Rightarrow \frac{s+t}{s+t+\left\|f_{1}, f_{2}, \ldots, f_{n}+f_{n}^{\prime}\right\|} & \geq \frac{t}{t+\left\|f_{1}, f_{2}, \ldots, f_{n}^{\prime}\right\|} \\
\Rightarrow \mu\left(f_{1}, f_{2}, \ldots, f_{n}+f_{n}^{\prime}, s+t\right) & \geq \min \left\{\mu\left(f_{1}, f_{2}, \ldots, f_{n}, s\right), \mu\left(f_{1}, f_{2}, \ldots, f_{n}^{\prime}, t\right)\right\} .
\end{aligned}
$$

(IFnN6) Clearly, $\lim _{t \rightarrow \infty} \mu\left(f_{1}, f_{2}, \ldots, f_{n}, t\right)=1$.

(IFnN7) Obviously, for all $t \in \mathbb{R}$ with $t \leq 0, \nu\left(f_{1}, f_{2}, \ldots, f_{n}, t\right)=1$.

(IFnN8) For all $t \in \mathbb{R}$ with $t<0$,

$$
\begin{aligned}
\nu\left(f_{1}, f_{2}, \ldots, f_{n}, t\right)=0 & \Leftrightarrow \frac{\left\|f_{1}, f_{2}, \ldots, f_{n}\right\|}{t+\left\|f_{1}, f_{2}, \ldots, f_{n}\right\|}=0 \Leftrightarrow\left\|f_{1}, f_{2}, \ldots, f_{n}\right\|=0 \\
& \Leftrightarrow f_{1}, f_{2}, \ldots, f_{n} \text { are linearly independent. }
\end{aligned}
$$

(IFnN9)

$$
\begin{aligned}
\nu\left(f_{1}, f_{2}, \ldots, f_{n}, t\right)=\frac{\left\|f_{1}, f_{2}, \ldots, f_{n}\right\|}{t+\left\|f_{1}, f_{2}, \ldots, f_{n}\right\|} & =\frac{\left\|f_{1}, f_{2}, \ldots, f_{n-1}\right\|}{t+\left\|f_{1}, f_{2}, \ldots, f_{n-1}\right\|} \\
& =\nu\left(f_{1}, f_{2}, \ldots, f_{n-1}, t\right)=\ldots
\end{aligned}
$$


Since $\left\|f_{1}, f_{2}, \ldots, f_{n}\right\|$ is invariant under any permutation of $f_{1}, f_{2}, \ldots, f_{n}$, it follows that $\nu\left(f_{1}, f_{2}, \ldots, f_{n}, t\right)$ is invariant under any permutation of $f_{1}, f_{2}, \ldots, f_{n}$.

(IFnN10) For $c \neq 0$ and $t>0$ we have

$$
\begin{aligned}
\nu\left(f_{1}, f_{2}, \ldots, c f_{n}, t\right) & =\frac{\left\|f_{1}, f_{2}, \ldots, c f_{n}\right\|}{t+\left\|f_{1}, f_{2}, \ldots, c f_{n}\right\|}=\frac{|c|\left\|f_{1}, f_{2}, \ldots, f_{n}\right\|}{t+|c|\left\|f_{1}, f_{2}, \ldots, f_{n}\right\|} \\
& =\frac{\left\|f_{1}, f_{2}, \ldots, f_{n}\right\|}{\frac{t}{|c|}+\left\|f_{1}, f_{2}, \ldots, f_{n}\right\|}=\nu\left(f_{1}, f_{2}, \ldots, f_{n}, \frac{t}{|c|}\right) .
\end{aligned}
$$

(IFnN11) We consider only the case when $s, t>0$ since other cases are obvious. Without loss of generality assume that

$$
\begin{gathered}
\nu\left(f_{1}, f_{2}, \ldots, f_{n}, s\right) \leq \nu\left(f_{1}, f_{2}, \ldots, f_{n}^{\prime}, t\right) \\
\Rightarrow \frac{\left\|f_{1}, f_{2}, \ldots, f_{n}\right\|}{s+\left\|f_{1}, f_{2}, \ldots, f_{n}\right\|} \leq \frac{\left\|f_{1}, f_{2}, \ldots, f_{n}^{\prime}\right\|}{t+\left\|f_{1}, f_{2}, \ldots, f_{n}^{\prime}\right\|} \\
\left\|f_{1}, f_{2}, \ldots, f_{n}\right\|\left(t+\| f_{1}, f_{2}, \ldots, f_{n}^{\prime}\right) \leq\left\|f_{1}, f_{2}, \ldots, f_{n}^{\prime}\right\|\left(s+\| f_{1}, f_{2}, \ldots, f_{n}\right) \\
\Rightarrow t\left\|f_{1}, f_{2}, \ldots, f_{n}\right\| \leq s\left\|f_{1}, f_{2}, \ldots, f_{n}^{\prime}\right\| .
\end{gathered}
$$

Now,

$$
\begin{aligned}
\frac{\left\|f_{1}, \ldots, f_{n}+f_{n}^{\prime}\right\|}{s+t+\left\|f_{1}, \ldots, f_{n}+f_{n}^{\prime}\right\|} & -\frac{\left\|f_{1}, \ldots, f_{n}^{\prime}\right\|}{s+t+\left\|f_{1}, \ldots, f_{n}^{\prime}\right\|} \\
& \leq \frac{\left\|f_{1}, \ldots, f_{n}\right\|+\left\|f_{1}, \ldots, f_{n}^{\prime}\right\|}{s+t+\left\|f_{1}, \ldots, f_{n}\right\|+\left\|f_{1}, \ldots, f_{n}^{\prime}\right\|} \\
& =\frac{t\left\|f_{1}, \ldots, f_{n}\right\|-s\left\|f_{1}, \ldots, f_{n}^{\prime}\right\|}{\left(s+t+\left\|f_{1}, \ldots, f_{n}\right\|+\left\|f_{1}, \ldots, f_{n}^{\prime}\right\|\right)\left(t+\left\|f_{1}, \ldots, f_{n}^{\prime}\right\|\right)}
\end{aligned}
$$

By $(3.1)$

$$
\frac{\left\|f_{1}, f_{2}, \ldots, f_{n}+f_{n}^{\prime}\right\|}{s+t+\left\|f_{1}, f_{2}, \ldots, f_{n}+f_{n}^{\prime}\right\|} \leq \frac{\left\|f_{1}, f_{2}, \ldots, f_{n}^{\prime}\right\|}{t+\left\|f_{1}, f_{2}, \ldots, f_{n}^{\prime}\right\|}
$$

Similarly,

$$
\begin{aligned}
\frac{\left\|f_{1}, f_{2}, \ldots, f_{n}+f_{n}^{\prime}\right\|}{s+t+\left\|f_{1}, f_{2}, \ldots, f_{n}+f_{n}^{\prime}\right\|} & \leq \frac{\left\|f_{1}, f_{2}, \ldots, f_{n}\right\|}{s+\left\|f_{1}, f_{2}, \ldots, f_{n}\right\|} \\
& \Rightarrow \nu\left(f_{1}, f_{2}, \ldots, f_{n}+f_{n}^{\prime}, s+t\right) \\
& \leq \max \left\{\nu\left(f_{1}, f_{2}, \ldots, f_{n}, s\right), \nu\left(f_{1}, f_{2}, \ldots, f_{n}^{\prime}, t\right)\right\}
\end{aligned}
$$

(IFnN12) Clearly, $\lim _{t \rightarrow \infty} \nu\left(f_{1}, f_{2}, \ldots, f_{n}, t\right)=0$.

Hence, $(F(X), \mu, \nu, *, \diamond)$ is an intuitionistic fuzzy $n$-normed linear space.

\section{Convergence and completeness}

In this section, we discuss some basic properties of sequences in an intuitionisitic 2-fuzzy $n$-normed linear space.

Definition 4.1. A sequence $\left\{f_{k}\right\}$ in an intuitionistic fuzzy $n$-normed linear space $(F(X), \mu, \nu, *, \diamond)$ is said to be:

(1) convergent to $f \in F(X)$ if for given $t>0,0<r<1$, there exists an integer $n_{0} \in \mathbb{N}$ such that $\mu\left(f_{k}-f, \omega_{2}, \omega_{3}, \ldots, \omega_{n}, t\right)>1-r$ and $\nu\left(f_{k}-f, \omega_{2}, \omega_{3}, \ldots, \omega_{n}, t\right)<r$ for all $n \geq n_{0}$ and for all $\omega_{2}, \omega_{3}, \ldots, \omega_{n} \in F(X)$; 
(2) a Cauchy sequence if for all $0<r<1, t>0$, there exists $n_{0} \in \mathbb{N}$ such that $\mu\left(f_{k}-f_{m}, \omega_{2}, \omega_{3}, \ldots, \omega_{n}, t\right)>1-\epsilon$ and $\nu\left(f_{k}-f_{m}, \omega_{2}, \omega_{3}, \ldots, \omega_{n}, t\right)<\epsilon$ for all $k, m \geq n_{0}$ and for all $\omega_{2}, \omega_{3}, \ldots, \omega_{n} \in F(X)$.

Definition 4.2. The intuitionistic fuzzy $n$-normed linear space $(F(X), \mu, \nu, *, \diamond)$ in which every Cauchy sequence converges is called complete.

Theorem 4.3. In an intuitionistic fuzzy n-normed linear space $(F(X), \mu, \nu, *, \diamond)$, a sequence $\left\{f_{k}\right\}$ converges to $f$ if and only if

$$
\lim _{n \rightarrow \infty} \mu\left(f_{k}-f, \omega_{2}, \omega_{3}, \ldots, \omega_{n}, t\right)=1, \quad \lim _{n \rightarrow \infty} \nu\left(f_{k}-f, \omega_{2}, \omega_{3}, \ldots, \omega_{n}, t\right)=0 .
$$

Proof. Fix $t>0$. Suppose $\left\{f_{k}\right\}$ converges to $f$. Then for a given $t>0,0<r<$ 1 , there exists an integer $n_{0} \in \mathbb{N}$ such that $\mu\left(f_{k}-f, \omega_{2}, \omega_{3}, \ldots, \omega_{n}, t\right)>1-r$ and $\nu\left(f_{k}-f, \omega_{2}, \omega_{3}, \ldots, \omega_{n}, t\right)<r$ for all $k \geq n_{0}$. Thus $1-\mu\left(f_{k}-f, \omega_{2}, \omega_{3}, \ldots, \omega_{n}, t\right)<r$ and $\nu\left(f_{k}-f, \omega_{2}, \omega_{3}, \ldots, \omega_{n}, t\right)<r$ and hence $\lim _{k \rightarrow \infty} \mu\left(f_{k}-f, \omega_{2}, \omega_{3}, \ldots, \omega_{n}, t\right)=1$ and $\lim _{k \rightarrow \infty} \nu\left(f_{k}-f, \omega_{2}, \omega_{3}, \ldots, \omega_{n}, t\right)=0$.

Conversely, if for each $t>0, \lim _{k \rightarrow \infty} \mu\left(f_{k}-f, \omega_{2}, \omega_{3}, \ldots, \omega_{n}, t\right)=1$ and $\lim _{k \rightarrow \infty} \nu\left(f_{k}-\right.$ $\left.f, \omega_{2}, \omega_{3}, \ldots, \omega_{n}, t\right)=0$, then for every $r, 0<r<1$, there exists an integer $n_{0} \in \mathbb{N}$ such that $1-\mu\left(f_{k}-f, \omega_{2}, \omega_{3}, \ldots, \omega_{n}, t\right)<r$ and $\nu\left(f_{k}-f, \omega_{2}, \omega_{3}, \ldots, \omega_{n}, t\right)<r$. Thus $\mu\left(f_{k}-f, \omega_{2}, \omega_{3}, \ldots, \omega_{n}, t\right)>1-r$ and $\nu\left(f_{k}-f, \omega_{2}, \omega_{3}, \ldots, \omega_{n}, t\right)<r$ for all $k \geq n_{0}$. Hence $\left\{f_{k}\right\}$ converges to $f$ in $(F(X), \mu, \nu, *, \diamond)$.

Theorem 4.4. If $*$ is continuous at the point $(1,1)$, and $\diamond$ is continuous at $(0,0)$, then the limit of a convergent sequence in an intuitionistic fuzzy n-normed linear space $(F(X), \mu, \nu, *, \diamond)$ is unique.

Proof. Suppose that $\left\{f_{k}\right\}$ converges to distinct $f$ and $h$ in $F(X)$. As $\operatorname{dim} F(X) \geq n$, there exist a linearly independent set of vectors $\left\{f-h, \omega_{2}, \omega_{3}, \ldots, \omega_{n}\right\}$ in $F(X)$. Now for $t \in \mathbb{R}$, we have

$$
\begin{aligned}
\mu\left(f-h, \omega_{2}, \omega_{3}, \ldots, \omega_{n}, 2 t\right)=\mu\left(f-f_{k}+f_{k}-h, \omega_{2}, \omega_{3}, \ldots, \omega_{n}, t+t\right) \\
\geq \mu\left(f-f_{k}, \omega_{2}, \omega_{3}, \ldots, \omega_{n}, t\right) * \mu\left(f_{k}-h, f_{1}, \omega_{2}, \omega_{3}, \ldots, \omega_{n}, t\right) .
\end{aligned}
$$

Taking limit to both sides and using the continuity of $t$-norm, we get

$$
\begin{aligned}
\lim _{2 t \rightarrow \infty} \mu\left(f-h, \omega_{2}, \omega_{3}, \ldots, \omega_{n}, t+t\right) \geq \lim _{t \rightarrow \infty} \mu\left(f-f_{k}, \omega_{2}, \omega_{3}, \ldots, \omega_{n}, t\right) \\
\quad * \lim _{t \rightarrow \infty} \mu\left(f_{k}-h, \omega_{2}, \omega_{3}, \ldots, \omega_{n}, t\right)=1 * 1=1 \text { for all } t>0 .
\end{aligned}
$$

Then $\left\{f-h, \omega_{2}, \omega_{3}, \ldots, \omega_{n}\right\}$ are linearly independent. This is a contradiction.

Similarly,

$$
\begin{array}{r}
\nu\left(f-h, \omega_{2}, \omega_{3}, \ldots, \omega_{n}, 2 t\right)=\nu\left(f-f_{k}+f_{k}-h, \omega_{2}, \omega_{3}, \ldots, \omega_{n}, t+t\right) \\
\leq \nu\left(f-f_{k}, \omega_{2}, \omega_{3}, \ldots, \omega_{n}, t\right) \diamond \nu\left(f_{k}-h, \omega_{2}, \omega_{3}, \ldots, \omega_{n}, t\right) .
\end{array}
$$

Taking limit to both sides and using continuity of $t$-conorm, we get

$$
\begin{aligned}
\lim _{2 t \rightarrow \infty} \nu\left(f-h, \omega_{2}, \omega_{3}, \ldots, \omega_{n}, t+t\right) \leq \lim _{s \rightarrow \infty} \nu\left(f-f_{k}, \omega_{2}, \omega_{3}, \ldots, \omega_{n}, t\right) \\
\diamond \lim _{t \rightarrow \infty} \nu\left(f_{k}-h, \omega_{2}, \omega_{3}, \ldots, \omega_{n}, t\right)=0 \diamond 0=0 \text { for all } t>0 .
\end{aligned}
$$

Then $\left\{f-h, \omega_{2}, \omega_{3}, \ldots, \omega_{n}\right\}$ are linearly independent. This is a contradiction. Thus $f-h=\underline{0}$, i.e. $f=h$.

Theorem 4.5. Let $(F(X), \mu, \nu, *, \diamond)$ be an intuitionistic fuzzy $n$-normed linear space such that $*$ is continuous at the point $(1,1)$, and $\diamond$ is continuous at $(0,0)$. If $\left\{f_{k}\right\}$ and $\left\{h_{k}\right\}$ are two sequences in $F(X)$ such that $\left\{f_{k}\right\}$ converges to $\{f\}$, and $\left\{h_{k}\right\}$ converges to $\{h\}$, then for $t>0$ :

(i) $\lim _{t \rightarrow \infty} \mu\left(\left(f_{k}+h_{k}\right)-(f+h), \omega_{2}, \omega_{3}, \ldots, \omega_{n}, 2 t\right)=1, \quad \lim _{t \rightarrow \infty} \nu\left(\left(f_{k}+h_{k}\right)-(f+\right.$ $\left.h), \omega_{2}, \omega_{3}, \ldots, \omega_{n}, 2 t\right)=0$; 
(ii) $\lim _{t \rightarrow \infty} \mu\left(c\left(f_{k}-f\right), \omega_{2}, \omega_{3}, \ldots, \omega_{n}, t\right)=1, \quad \lim _{t \rightarrow \infty} \nu\left(c\left(f_{k}-f\right), \omega_{2}, \ldots, \omega_{n}, t\right)$ $=0$ for $c \in \mathbb{K}$ with $c \neq 0$.

Proof. (i) We have the following

$$
\begin{aligned}
& \mu\left(\left(f_{k}+h_{k}\right)-(f+h), \omega_{2}, \omega_{3}, \ldots, \omega_{n}, 2 t\right) \\
& =\mu\left(\left(f_{k}-f\right)+\left(h_{k}-h\right), \omega_{2}, \omega_{3}, \ldots, \omega_{n}, t+t\right) \\
& \geq \mu\left(f_{k}-f, \omega_{2}, \omega_{3}, \ldots, \omega_{n}, t\right) * \mu\left(h_{k}-h, \omega_{2}, \omega_{3}, \ldots, \omega_{n}, t\right)=1 * 1=1 .
\end{aligned}
$$

Taking limit and using the continuity of the $t$-norm, we get

$$
\begin{aligned}
\lim _{2 t \rightarrow \infty} \mu\left(\left(f_{k}+h_{k}\right)\right. & \left.-(f+h), \omega_{2}, \omega_{3}, \ldots, \omega_{n}, 2 t\right) \\
& =\lim _{2 t \rightarrow \infty} \mu\left(\left(f_{k}-f\right)+\left(h_{k}-h\right), \omega_{2}, \omega_{3}, \ldots, \omega_{n}, t+t\right) \\
& \geq \lim _{t \rightarrow \infty} \mu\left(f_{k}-f, \omega_{2}, \omega_{3}, \ldots, \omega_{n}, t\right) \\
& * \lim _{t \rightarrow \infty} \mu\left(h_{k}-h, \omega_{2}, \omega_{3}, \ldots, \omega_{n}, t\right)=1 * 1=1 .
\end{aligned}
$$

In a similar way we have

$$
\begin{aligned}
\nu\left(\left(f_{k}+h_{k}\right)\right. & \left.-(f+h), \omega_{2}, \omega_{3}, \ldots, \omega_{n}, 2 t\right) \\
& =\nu\left(\left(f_{k}-f\right)+\left(h_{k}-h\right), \omega_{2}, \omega_{3}, \ldots, \omega_{n}, t+t\right) \\
& \leq \nu\left(f_{k}-f, \omega_{2}, \omega_{3}, \ldots, \omega_{n}, t\right) \\
& * \nu\left(h_{k}-h, \omega_{2}, \omega_{3}, \ldots, \omega_{n}, t\right)=1 * 1=1 .
\end{aligned}
$$

Taking limit and using the continuity of $t$-conorm, we get

$$
\begin{aligned}
\lim _{2 t \rightarrow \infty} \nu\left(\left(f_{k}+h_{k}\right)\right. & \left.-(f+h), \omega_{2}, \omega_{3}, \ldots, \omega_{n}, 2 t\right) \\
& =\lim _{2 t \rightarrow \infty} \nu\left(\left(f_{k}-f\right)+\left(h_{k}-h\right), \omega_{2}, \omega_{3}, \ldots, \omega_{n}, t+t\right) \\
& \leq \lim _{t \rightarrow \infty} \nu\left(f_{k}-f, \omega_{2}, \omega_{3}, \ldots, \omega_{n}, t\right) \\
& * \lim _{t \rightarrow \infty} \nu\left(h_{k}-h, \omega_{2}, \omega_{3}, \ldots, \omega_{n}, t\right)=0 \diamond 0=0 .
\end{aligned}
$$

(ii) First, $\mu\left(c\left(f_{k}-f\right), \omega_{2}, \omega_{3}, \ldots, \omega_{n}, t\right)=\mu\left(f_{k}-f, \omega_{2}, \omega_{3}, \ldots, \omega_{n}, \frac{t}{|c|}\right)$.

Let $s=\frac{t}{|c|}$ and taking limit of the above equation, we get

$$
\lim _{s \rightarrow \infty} \mu\left(f_{k}-f, \omega_{2}, \omega_{3}, \ldots, \omega_{n}, s\right)=1 .
$$

Again,

$$
\nu\left(c\left(f_{k}-f\right), \omega_{2}, \omega_{3}, \ldots, \omega_{n}, t\right)=\nu\left(f_{k}-f, \omega_{2}, \omega_{3}, \ldots, \omega_{n}, \frac{t}{|c|}\right) .
$$

Let $s=\frac{t}{|c|}$ and taking limit of the above equation, we get

$$
\lim _{s \rightarrow \infty} \nu\left(f_{k}-f, \omega_{2}, \omega_{3}, \ldots, \omega_{n}, s\right)=0 .
$$

Theorem 4.6. In an intuitionistic fuzzy n-normed linear space $(F(X), \mu, \nu, *, \diamond)$, every convergent sequence is a Cauchy sequence.

Proof. Let $\left\{f_{k}\right\}$ be a convergent sequence in $(F(X), \mu, \nu, *, \diamond)$ converging to $f \in F(X)$. Let $t>0, \epsilon \in(0,1)$. Choose $r \in(0,1)$ such that $(1-r) *(1-r)>1-\epsilon$ and $r \diamond r<\epsilon$. Since $\left\{f_{k}\right\}$ converges to $f$, there exists an integer $n_{0} \in \mathbb{N}$ such that $\mu\left(f_{k}-f, \omega_{2}, \omega_{3}, \ldots, \omega_{n}, \frac{t}{2}\right)>$ 
$1-r$ and $\nu\left(f_{k}-f, \omega_{2}, \omega_{3}, \ldots, \omega_{n}, \frac{t}{2}\right)<r$ for all $k \geq n_{0}$. Now

$$
\begin{aligned}
\mu\left(f_{k}-f_{m}, \omega_{2}, \omega_{3}, \ldots, \omega_{n}, t\right)=\mu\left(f_{k}-f+f-f_{m}, \omega_{2}, \omega_{3}, \ldots, \omega_{n}, \frac{t}{2}+\frac{t}{2}\right) \\
\quad \geq \mu\left(f_{k}-f, \omega_{2}, \omega_{3}, \ldots, \omega_{n}, \frac{t}{2}\right) * \mu\left(f-f_{m}, \omega_{2}, \omega_{3}, \ldots, \omega_{n}, \frac{t}{2}\right) \\
>(1-r) *(1-r), \text { for all } k, m \geq n_{0} \\
>(1-\epsilon), \text { for all } k, m \geq n_{0},
\end{aligned}
$$

and

$$
\begin{aligned}
\nu\left(f_{k}-f_{m}, \omega_{2}, \omega_{3}, \ldots, \omega_{n}, t\right)=\nu\left(f_{k}-f+f-f_{m}, \omega_{2}, \omega_{3}, \ldots, \omega_{n}, \frac{t}{2}+\frac{t}{2}\right) \\
\quad \leq \nu\left(f_{k}-f, \omega_{2}, \omega_{3}, \ldots, \omega_{n}, \frac{t}{2}\right) \diamond \nu\left(f-f_{m}, \omega_{2}, \omega_{3}, \ldots, \omega_{n}, \frac{t}{2}\right) \\
<r \diamond r, \text { for all } k, m \geq n_{0} \\
<\epsilon, \text { for all } k, m \geq n_{0} .
\end{aligned}
$$

Therefore, $\left\{f_{k}\right\}$ is a Cauchy sequence in $(F(X), \mu, \nu, *, \diamond)$.

Remark 4.7. The converse of Theorem 4.6 is not necessarily true. It is verified by the following example.

Example 4.8. Let $(F(X),\|\cdot, \ldots, \cdot\|)$ be an $n$-normed linear space and define $a * b=$ $\min \{a, b\}$ and $a \diamond b=\max \{a, b\}$ for all $a, b \in[0,1], t>0 . \mu\left(f_{1}, f_{2}, \ldots, f_{n}, t\right)=\frac{t}{t+\left\|f_{1}, f_{2}, \ldots, f_{n}\right\|}$ and $\nu\left(f_{1}, f_{2}, \ldots, f_{n}, t\right)=\frac{\left\|f_{1}, f_{2}, \ldots, f_{n}\right\|}{t+\left\|f_{1}, f_{2}, \ldots, f_{n}\right\|}$. If

$$
A=\left\{F(X), \mu\left(f_{1}, f_{2}, \ldots, f_{n}, t\right), \nu\left(f_{1}, f_{2}, \ldots, f_{n}, t\right): f_{1}, f_{2}, \ldots, f_{n} \in[F(X)]^{n}\right\},
$$

then by Example 3.3, $(F(X), \mu, \nu, *, \diamond)$ is an intuitionistic fuzzy $n$-normed linear space. Let $\left\{f_{n}\right\}$ be a sequence in $(F(X), \mu, \nu, *, \diamond)$. Then

(i) $\left\{f_{n}\right\}$ is a Cauchy sequence in $(F(X),\|\cdot, \ldots, \cdot\|)$ if and only if $\left\{f_{n}\right\}$ is a Cauchy sequence in intuitionistic fuzzy $n$-normed linear space $(F(X), \mu, \nu, *, \diamond)$.

(ii) $\left\{f_{n}\right\}$ is a convergent sequence in $(F(X),\|\cdot, \ldots, \cdot\|)$ if and only if $\left\{f_{n}\right\}$ is a convergent sequence in intuitionistic fuzzy $n$-normed linear space $(F(X), \mu, \nu, *, \diamond)$.

(i) $\left\{f_{n}\right\}$ is a Cauchy sequence in $(F(X),\|\cdot, \ldots, \cdot\|)$

$$
\begin{aligned}
\Leftrightarrow & \lim _{n, k \rightarrow \infty}\left\|f_{1}, f_{2}, \ldots, f_{n}-f_{k}\right\|=0 . \\
\Leftrightarrow & \lim _{n, k \rightarrow \infty} \mu\left(f_{1}, f_{2}, \ldots, f_{n}-f_{k}, t\right)=\lim _{n, k \rightarrow \infty} \frac{t}{t+\left\|f_{1}, f_{2}, \ldots, f_{n}-f_{k}\right\|}=1 \\
& \text { and } \lim _{n, k \rightarrow \infty} \nu\left(f_{1}, f_{2}, \ldots, f_{n}-f_{k}, t\right)=\lim _{n, k \rightarrow \infty} \frac{\left\|f_{1}, f_{2}, \ldots, f_{n}-f_{k}\right\|}{t+\left\|f_{1}, f_{2}, \ldots, f_{n}-f_{k}\right\|}=0 . \\
\Leftrightarrow & \mu\left(f_{1}, f_{2}, \ldots, f_{n}-f_{k}, t\right)>1-r \text { and } \nu\left(f_{1}, f_{2}, \ldots, f_{n}-f_{k}, t\right)<r \\
& \quad \text { for each } r \in(0,1), \text { for all } n, k \geq n_{0} . \\
\Leftrightarrow & \left\{f_{n}\right\} \text { is a Cauchy sequence in }(F(X), \mu, \nu, *, \diamond) .
\end{aligned}
$$


(ii) $\left\{f_{n}\right\}$ is a convergent sequence in $(F(X),\|\cdot, \ldots, \cdot\|)$

$$
\begin{aligned}
\Leftrightarrow & \lim _{n \rightarrow \infty}\left\|f_{1}, f_{2}, \ldots, f_{n}-f\right\|=0 . \\
\Leftrightarrow & \lim _{n \rightarrow \infty} \mu\left(f_{1}, f_{2}, \ldots, f_{n}-f, t\right)=\lim _{n \rightarrow \infty} \frac{t}{t+\left\|f_{1}, f_{2}, \ldots, f_{n}-f\right\|}=1 \\
& \text { and } \lim _{n \rightarrow \infty} \nu\left(f_{1}, f_{2}, \ldots, f_{n}-f, t\right)=\lim _{n \rightarrow \infty} \frac{\left\|f_{1}, f_{2}, \ldots, f_{n}-f\right\|}{t+\left\|f_{1}, f_{2}, \ldots, f_{n}-f\right\|}=0 . \\
\Leftrightarrow & \mu\left(f_{1}, f_{2}, \ldots, f_{n}-f, t\right)>1-r \text { and } \nu\left(f_{1}, f_{2}, \ldots, f_{n}-f, t\right)<r, \\
& \quad \text { for each } r \in(0,1), \text { for all } n \geq n_{0} . \\
\Leftrightarrow & \left\{f_{n}\right\} \text { is a convergent sequence in }(F(X), \mu, \nu, *, \diamond) .
\end{aligned}
$$

Thus if there exists an 2-fuzzy $n$-normed linear space $(F(X),\|\cdot, \ldots, \cdot\|)$ which is not complete, then the IFnN induced by such a crisp $2-n$-norm $\|\cdot, \ldots, \cdot\|$ on an incomplete $2-n$ normed linear space $F(X)$ is an incomplete intuitionistic fuzzy $n$-normed linear space.

Theorem 4.9. Let $(F(X), \mu, \nu, *, \diamond)$ be an intuitionistic fuzzy $n$-normed linear space with underlying $t$-norm being continuous at $(1,1)$, and $t$-conorm is continuous at $(0,0)$ such that every Cauchy sequence in $(F(X), \mu, \nu, *, \diamond)$ has a convergent subsequence. Then $(F(X), \mu, \nu, *, \diamond)$ is a complete intuitionistic fuzzy n-normed linear space.

Proof. Let $\left\{f_{k}\right\}$ is a Cauchy sequence in $(F(X), \mu, \nu, *, \diamond)$ and let $\left\{f_{k_{s}}\right\}$ be a subsequence of $\left\{f_{k}\right\}$ that converge to $f$. We prove that $\left\{f_{k}\right\}$ converges to $f$. Let $t>0, \epsilon \in(0,1)$ and choose $r \in(0,1)$ be such that $(1-r) *(1-r)>1-\epsilon$ and $r \diamond r<\epsilon$. Since $\left\{f_{k}\right\}$ is a Cauchy sequence, there exist an integer $n_{0} \in \mathbb{N}$ such that $\mu\left(f_{k}-f_{m}, \omega_{2}, \omega_{3}, \ldots, \omega_{n}, \frac{t}{2}\right)>1-r$ and $\nu\left(f_{k}-f_{m}, \omega_{2}, \omega_{3}, \ldots, \omega_{n}, \frac{t}{2}\right)<r$, for all $k, m \geq n_{0}$. Since $\left\{f_{k_{s}}\right\}$ converges to $f$, there is a positive integer $i_{s}>n_{0}$ such that $\mu\left(f_{i_{s}}-f, \omega_{2}, \omega_{3}, \ldots, \omega_{n}, \frac{t}{2}\right)>1-r$ and $\nu\left(f_{i_{s}}-f, \omega_{2}, \omega_{3}, \ldots, \omega_{n}, \frac{t}{2}\right)<r$. Now

$$
\begin{aligned}
\mu\left(f_{k}-f, \omega_{2}, \omega_{3}, \ldots, \omega_{n}, t\right)=\mu\left(f_{k}-f_{i_{s}}+f_{i_{s}}-f, \omega_{2}, \omega_{3}, \ldots, \omega_{n}, \frac{t}{2}+\frac{t}{2}\right) \\
\geq \mu\left(f_{k}-f_{i_{s}}, \omega_{2}, \omega_{3}, \ldots, \omega_{n}, \frac{t}{2}\right) * \mu\left(f_{i_{s}}-f, \omega_{2}, \omega_{3}, \ldots, \omega_{n}, \frac{t}{2}\right) \\
>(1-r) *(1-r)>1-\epsilon,
\end{aligned}
$$

and

$$
\begin{aligned}
\nu\left(f_{k}-f, \omega_{2}, \omega_{3}, \ldots, \omega_{n}, t\right)=\nu\left(f_{k}-f_{i_{s}}+f_{i_{s}}-f, \omega_{2}, \omega_{3}, \ldots, \omega_{n}, \frac{t}{2}+\frac{t}{2}\right) \\
\geq \nu\left(f_{k}-f_{i_{s}}, \omega_{2}, \omega_{3}, \ldots, \omega_{n}, \frac{t}{2}\right) \diamond \nu\left(f_{i_{s}}-f, \omega_{2}, \omega_{3}, \ldots, \omega_{n}, \frac{t}{2}\right) \\
<r \diamond r<\epsilon .
\end{aligned}
$$

Therefore, $\left\{f_{k}\right\}$ converges to $f$ in intuitionistic fuzzy $n$-normed linear space $(F(X), \mu, \nu, *, \diamond)$ and hence it is complete.

As a consequence of [15, Theorem 3.1], we introduce an interesting notion of ascending family of $\alpha$-n-norms corresponding to the intuitionisitic 2 -fuzzy $n$-norm in the following theorem.

Theorem 4.10. Let $(F(X), \mu, \nu, *, \diamond)$ be an intuitionistic fuzzy $n$-normed linear space such that:

(i) $a * a=a, a \diamond a=a$ for all $a \in[0,1]$;

(ii) For all $t \in \mathbb{R}$ with $t>0, \mu\left(f_{1}, f_{2}, \ldots, f_{n}, t\right)>0$ implies $f_{1}, f_{2}, \ldots, f_{n}$ are linearly independent;

(iii) For all $t \in \mathbb{R}$ with $t>0, \nu\left(f_{1}, f_{2}, \ldots, f_{n}, t\right)<1$ implies $f_{1}, f_{2}, \ldots, f_{n}$ are linearly independent. 
Define

$$
\begin{aligned}
& \left\|f_{1}, f_{2}, \ldots, f_{n}\right\|_{\alpha}=\inf \left\{t: \mu\left(f_{1}, f_{2}, \ldots, f_{n}, t\right) \geq \alpha, \alpha \in(0,1)\right\} \text { and } \\
& \left\|f_{1}, f_{2}, \ldots, f_{n}\right\|_{\alpha}=\sup \left\{t: \nu\left(f_{1}, f_{2}, \ldots, f_{n}, t\right) \leq 1-\alpha, \alpha \in(0,1)\right\}
\end{aligned}
$$

Then $\left\{\left\|f_{1}, f_{2}, \ldots, f_{n}\right\|_{\alpha}: \alpha \in(0,1)\right\}$ is an ascending family of n-norms on $F(X)$.

Proof. Let $\alpha \in(0,1)$. We show that $\left\|f_{1}, f_{2}, \ldots, f_{n-1}, f_{n}\right\|_{\alpha}$ is an $n$-norm on $F(X)$.

(i) Let $\left\|f_{1}, f_{2}, \ldots, f_{n}\right\|_{\alpha}=0$. This implies $\inf \left\{t: \mu\left(f_{1}, f_{2}, \ldots, f_{n}, t\right) \geq \alpha, \alpha \in(0,1)\right\}=0$ and $\sup \left\{t: \nu\left(f_{1}, f_{2}, \ldots, f_{n}, t\right) \leq 1-\alpha, \alpha \in(0,1)\right\}=0$. Then for all $\alpha \in(0,1)$, $\mu\left(f_{1}, f_{2}, \ldots, f_{n}, t\right) \geq \alpha>0$ and $\nu\left(f_{1}, f_{2}, \ldots, f_{n}, t\right) \leq 1-\alpha<1$ which implies that $f_{1}, f_{2}, \ldots, f_{n}$ are linearly independent.

Conversely, assume that $f_{1}, \ldots, f_{n}$ are linearly independent. This implies $\mu\left(f_{1}, \ldots, f_{n}, t\right)=$ 1 and $\nu\left(f_{1}, \ldots, f_{n}, t\right)=0$ for all $t>0$, we have $\inf \left\{t: \mu\left(f_{1}, \ldots, f_{n}, t\right) \geq \alpha, \alpha \in(0,1)\right\}=0$ and $\sup \left\{t: \nu\left(f_{1}, f_{2}, \ldots, f_{n}, t\right) \leq 1-\alpha, \alpha \in(0,1)\right\}=0$ this implies $\left\|f_{1}, f_{2}, \ldots, f_{n}\right\|_{\alpha}=0$.

(ii) Since $\mu\left(f_{1}, f_{2}, \ldots, f_{n}, t\right)$ and $\nu\left(f_{1}, f_{2}, \ldots, f_{n}, t\right)$ are invariant under any permutation it follows that $\left\|f_{1}, f_{2}, \ldots, f_{n}\right\|_{\alpha}$ is also invariant under any permutation.

(iii) If $c \neq 0$, then

$$
\begin{aligned}
\left\|f_{1}, f_{2}, \ldots, c f_{n}\right\|_{\alpha} & =\inf \left\{s: \mu\left(f_{1}, f_{2}, \ldots, c f_{n}, s\right) \geq \alpha, \alpha \in(0,1)\right\} \\
& =\inf \left\{s: \mu\left(f_{1}, f_{2}, \ldots, f_{n}, \frac{s}{|c|}\right) \geq \alpha, \alpha \in(0,1)\right\} .
\end{aligned}
$$

Similarly,

$$
\begin{aligned}
\left\|f_{1}, f_{2}, \ldots, c f_{n}\right\|_{\alpha} & =\sup \left\{s: \nu\left(f_{1}, f_{2}, \ldots, c f_{n}, s\right) \leq 1-\alpha, \alpha \in(0,1)\right\} \\
& =\sup \left\{s: \nu\left(f_{1}, f_{2}, \ldots, f_{n}, \frac{s}{|c|}\right) \leq 1-\alpha, \alpha \in(0,1)\right\} .
\end{aligned}
$$

Let $t=\frac{s}{|c|}$, then we have

$$
\begin{aligned}
\left\|f_{1}, f_{2}, \ldots, c f_{n}\right\|_{\alpha} & =\inf \left\{|c| t: \mu\left(f_{1}, f_{2}, \ldots, f_{n}, t\right) \geq \alpha, \alpha \in(0,1)\right\} \\
& =|c| \inf \left\{t: \mu\left(f_{1}, f_{2}, \ldots, f_{n}, t\right) \geq \alpha, \alpha \in(0,1)\right\} \\
& =|c|\left\|f_{1}, f_{2}, \ldots, f_{n}\right\|_{\alpha}
\end{aligned}
$$

and

$$
\begin{aligned}
\left\|f_{1}, f_{2}, \ldots, c f_{n}\right\|_{\alpha} & =\sup \left\{|c| t: \nu\left(f_{1}, f_{2}, \ldots, f_{n}, t\right) \leq 1-\alpha, \alpha \in(0,1)\right\} \\
& =|c| \sup \left\{t: \nu\left(f_{1}, f_{2}, \ldots, f_{n}, t\right) \leq 1-\alpha, \alpha \in(0,1)\right\} \\
& =|c|\left\|f_{1}, f_{2}, \ldots, f_{n}\right\|_{\alpha} .
\end{aligned}
$$

If $c=0$, then

$$
\begin{aligned}
\left\|f_{1}, f_{2}, \ldots, c f_{n}\right\|_{\alpha} & =\left\|f_{1}, f_{2}, \ldots, 0\right\|_{\alpha}=0 \\
& =0\left\|f_{1}, f_{2}, \ldots, f_{n}\right\|_{\alpha}=|c|\left\|f_{1}, f_{2}, \ldots, f_{n}\right\|_{\alpha} \text { for all } c \in \mathbb{R} .
\end{aligned}
$$

(iv) We have

$$
\begin{aligned}
\left\|f_{1}, f_{2}, \ldots, f_{n}\right\|_{\alpha} & +\left\|f_{1}, f_{2}, \ldots, f_{n}^{\prime}\right\|_{\alpha}=\inf \left\{s: \mu\left(f_{1}, f_{2}, \ldots, f_{n}, s\right) \geq \alpha\right\} \\
& +\inf \left\{t: \mu\left(f_{1}, f_{2}, \ldots, f_{n}^{\prime}, t\right) \geq \alpha\right\} \\
& \geq \inf \left\{s+t: \mu\left(f_{1}, f_{2}, \ldots, f_{n}, s\right) \geq \alpha, \mu\left(f_{1}, f_{2}, \ldots, f_{n}^{\prime}, t\right) \geq \alpha\right\} \\
& \geq \inf \left\{s+t: \mu\left(f_{1}, f_{2}, \ldots, f_{n}, s\right) * \mu\left(f_{1}, f_{2}, \ldots, f_{n}^{\prime}, t\right) \geq \alpha * \alpha\right\} \\
& \geq \inf \left\{s+t: \mu\left(f_{1}, f_{2}, \ldots, f_{n}+f_{n}^{\prime}, s+t\right) \geq \alpha\right\} \\
& =\left\|f_{1}, f_{2}, \ldots, f_{n}+f_{n}^{\prime}\right\|_{\alpha} .
\end{aligned}
$$


Again

$$
\begin{aligned}
\| f_{1}, f_{2}, \ldots, & f_{n}\left\|_{\alpha}+\right\| f_{1}, f_{2}, \ldots, f_{n}^{\prime} \|_{\alpha}=\sup \left\{s: \nu\left(f_{1}, f_{2}, \ldots, f_{n}, s\right) \leq 1-\alpha\right\} \\
& +\sup \left\{t: \nu\left(f_{1}, f_{2}, \ldots, f_{n}^{\prime}, t\right) \leq 1-\alpha\right\} \\
& \geq \sup \left\{s+t: \nu\left(f_{1}, f_{2}, \ldots, f_{n}, s\right) \leq 1-\alpha, \nu\left(f_{1}, f_{2}, \ldots, f_{n}^{\prime}, t\right) \leq 1-\alpha\right\} \\
& \geq \sup \left\{s+t: \nu\left(f_{1}, f_{2}, \ldots, f_{n}, s\right) \diamond \nu\left(f_{1}, f_{2}, \ldots, f_{n}^{\prime}, t\right) \leq 1-\alpha \diamond 1-\alpha\right\} \\
& =\left\|f_{1}, f_{2}, \ldots, f_{n}+f_{n}^{\prime}\right\|_{\alpha} .
\end{aligned}
$$

Therefore,

$$
\left\|f_{1}, f_{2}, \ldots, f_{n}+f_{n}^{\prime}\right\|_{\alpha} \leq\left\|f_{1}, f_{2}, \ldots, f_{n}\right\|_{\alpha}+\left\|f_{1}, f_{2}, \ldots, f_{n}^{\prime}\right\|_{\alpha} .
$$

Thus $\left\|f_{1}, f_{2}, \ldots, f_{n}\right\|_{\alpha}$ is an $n$-norm on $F(X)$.

Let $0<\alpha_{1}<\alpha_{2}<1$. Then

$$
\begin{aligned}
& \left\|f_{1}, f_{2}, \ldots, f_{n}\right\|_{\alpha_{1}} \inf \left\{t: \mu\left(f_{1}, f_{2}, \ldots, f_{n}, t\right) \geq \alpha_{1}\right\} \text { and } \\
& \left\|f_{1}, f_{2}, \ldots, f_{n}\right\|_{\alpha_{2}} \inf \left\{t: \mu\left(f_{1}, f_{2}, \ldots, f_{n}, t\right) \geq \alpha_{2}\right\} . \\
& \left\|f_{1}, f_{2}, \ldots, f_{n}\right\|_{\alpha_{1}} \sup \left\{t: \nu\left(f_{1}, f_{2}, \ldots, f_{n}, t\right) \leq 1-\alpha_{1}\right\} \text { and } \\
& \left\|f_{1}, f_{2}, \ldots, f_{n}\right\|_{\alpha_{2}} \sup \left\{t: \nu\left(f_{1}, f_{2}, \ldots, f_{n}, t\right) \leq 1-\alpha_{2}\right\} .
\end{aligned}
$$

Since $\alpha_{1}<\alpha_{2}$

$$
\left\{t: \mu\left(f_{1}, f_{2}, \ldots, f_{n}, t\right) \geq \alpha_{2}\right\} \subset\left\{t: \mu\left(f_{1}, f_{2}, \ldots, f_{n}, t\right) \geq \alpha_{1}\right\}
$$

implies

$$
\inf \left\{t: \mu\left(f_{1}, f_{2}, \ldots, f_{n}, t\right) \geq \alpha_{2}\right\} \geq \inf \left\{t: \mu\left(f_{1}, f_{2}, \ldots, f_{n}, t\right) \geq \alpha_{1}\right\}
$$

and

implies

$$
\left\{t: \nu\left(f_{1}, f_{2}, \ldots, f_{n}, t\right) \leq 1-\alpha_{2}\right\} \subset\left\{t: \mu\left(f_{1}, f_{2}, \ldots, f_{n}, t\right) \leq 1-\alpha_{1}\right\}
$$

$$
\sup \left\{t: \nu\left(f_{1}, \ldots, f_{n}, t\right) \leq 1-\alpha_{2}\right\} \geq \sup \left\{t: \mu\left(f_{1}, \ldots, f_{n}, t\right) \leq 1-\alpha_{1}\right\} .
$$

Therefore,

$$
\left\|f_{1}, f_{2}, \ldots, f_{n}\right\|_{\alpha_{2}} \geq\left\|f_{1}, f_{2}, \ldots, f_{n}\right\|_{\alpha_{1}} .
$$

Hence $\left\{\left\|f_{1}, f_{2}, \ldots, f_{n}\right\|_{\alpha}: \alpha \in(0,1)\right\}$ is an ascending family of $\alpha$-n-norms on $F(X)$.

The $n$-norms described in the previous theorem are called $\alpha$-n-norms on $F(X)$ corresponding to the intuitionistic 2-fuzzy n-norms on $X$.

\section{References}

[1] K.T. Atanassov, Intuitionistic fuzzy sets, Fuzzy Sets Syst. 20 (1), 87-96, 1986.

[2] K.T. Atanassov, More on intuitionistic fuzzy sets, Fuzzy Sets Syst. 33 (1), 37-45, 1989.

[3] T. Bag and S.K. Samanta, Finite dimensional fuzzy normed linear spaces, J. Fuzzy Math. 11 (3), 687-705, 2003.

[4] T. Bag and S.K. Samanta, Finite dimensional intuitionistic fuzzy normed linear spaces, Ann. Fuzzy Math. Inform. 6 (2), 45-57, 2013.

[5] D. Çoker, An introduction to intuitionistic fuzzy topological spaces, Fuzzy Sets Syst. 88 (1), 81-89, 1997.

[6] S. Gähler, Lineare 2-normierte Räume, Math. Nachr. 28 (1-2), 1-43, 1964.

[7] S. Gähler, Untersuchungen über verallgemeinerte m-metrische Räume, Math. Nachr. 40 (1-3), 165-189, 1969.

[8] R. Giles, A computer program for fuzzy reasoning, Fuzzy Sets Syst. 4 (3), 221-234, 1980. 
[9] H. Gunawan and M. Mashadi, On n-normed spaces, Internat. J. Math. Math. Sci. 27 (10), 631-639, 2001.

[10] L. Hong and J.-Q. Sun, Bifurcations of fuzzy nonlinear dynamical systems, Commun. Nonlinear Sci. Numer. Simul. 11 (1), 1-12, 2006.

[11] S.S. Kim and Y.J. Cho, Strict convexity in linear n-normed spaces, Demonstratio Math. 29 (4), 739-744, 1996.

[12] A. Misiak, n-inner product spaces, Math. Nachr. 140 (1), 299-319, 1989.

[13] M. Mursaleen and Q.M. Danish Lohani, Intuitionistic fuzzy 2-normed space and some related concepts, Chaos Solitons Fract. 42 (1), 224-234, 2009.

[14] A.L. Narayanan and S. Vijayabalaji, Fuzzy n-normed linear spaces, Internat. J. Math. Math. Sci. 24, 3963-3977, 2005.

[15] J.H. Park, Intuitionistic fuzzy metric spaces, Chaos Solitons Fract. 22 (5), 1039-1046, 2004.

[16] C. Park and C. Alaca, An introduction to 2-fuzzy n-normed linear spaces and a new perspective to the Mazur-Ulam problem, J. Inequal. Appl. 14, 2012.

[17] M.H.M. Rashid and Lj.D.R. Kočinac, Ideal convergence in 2-fuzzy 2-normed spaces, Hacet. J. Math. Stat. 46 (1), 145-159, 2017.

[18] R. Saadati and J.H. Park, On the intuitionistic fuzzy topological spaces, Chaos Solitons Fract. 27 (2), 331-344, 2006.

[19] B. Schweizer and A. Sklar, Statistical metric spaces, Pacific J. Math. 10 (1), 313-334, 1960.

[20] R.M. Somasundaram and T. Beaula, Some aspects of 2-fuzzy 2-normed linear spaces, Bull. Malays. Math. Sci. Soc. 32 (2), 211-221, 2009.

[21] L.A. Zadeh, Fuzzy sets, Inform. and Control 8 (3), 338-353, 1965. 